\title{
Static deformation of two welded monoclinic elastic half-spaces due to a long inclined strike-slip fault
}

\author{
Anil Kumar, Sarvajit Singh* and Jagdish Singh \\ Department of Mathematics, Maharshi Dayanand University, Rohtak 124 001, India. \\ *e-mail: s-j_singh@yahoo.com
}

Static deformation of two monoclinic elastic half-spaces in welded contact due to a long inclined strike-slip fault situated in one of the half-spaces is studied analytically and numerically. Closedform algebraic expressions for the displacement at any point of the medium are obtained. The variation of the displacement at the interface with the horizontal distance from the fault is studied. The effect of anisotropy on the displacement field is examined. It is found that while the anisotropy of the source half-space has a significant effect on the displacement at the interface, the anisotropy of the other half-space has only a marginal effect.

\section{Introduction}

The deformation of an isotropic elastic half-space by a long strike-slip fault has been investigated very extensively (see e.g., Maruyama 1966; Savage 1980). The deformation of two welded isotropic half-spaces by a long strike-slip fault has been studied, among others, by Sharma et al (1991) and Rani and Singh (1993). Pan (1989) formulated the problem of the deformation of a transversely isotropic multilayered half-space by a dislocation source in terms of layer matrices. Garg et al (1996) obtained an analytical solution for the deformation of an orthotropic layered half-space caused by a long strike-slip fault. Ting (1995) derived the Green's functions for a line force and a screw dislocation for the antiplane deformation of a monoclinic elastic medium consisting of a single half-space or two half-spaces in welded contact. Singh et al (2001) obtained closed-form analytical expression for the horizontal displacement caused by a long inclined strike-slip fault located in a monoclinic elastic halfspace.

In the present study, we use the results of Ting (1995) to obtain closed-form analytical expressions for the horizontal displacement due to a long inclined strike-slip fault situated in a monoclinic elastic half-space in welded contact with another monoclinic elastic half-space. The fault is of infinite length in the strike-direction and of finite width in the down-dip-direction. Monoclinic symmetry is the symmetry of two sets of non-orthogonal parallel cracks, where the plane of symmetry is perpendicular to the lines of intersection of the two sets of crack faces. Monoclinic symmetry of systems of cracks may be found near the surface of the earth where lithostatic pressures have not closed cracks perpendicular to the maximum compressional stress (Crampin 1989).

\section{Basic equations}

The generalized Hooke's law for an anisotropic elastic medium may be expressed in the form

$$
\begin{aligned}
\tau_{i j} & =C_{i j k s} u_{k, s}, \\
\left(u_{k, s}\right. & \left.=\partial u_{k} / \partial x_{s}\right),
\end{aligned}
$$

where $\tau_{i j}$ is the stress tensor, $u_{k}$ is the displacement vector and $C_{i j k s}$ are the elastic stiffnesses satisfying the symmetry relations

$$
C_{i j k s}=C_{j i k s}=C_{k s i j} .
$$

Summation over repeated indices is understood.

Keywords. Monoclinic elastic media; static deformation; strike-slip fault; welded half-spaces. 
In the absence of body forces, the equations of equilibrium can be expressed in the form

$$
C_{i j k s} u_{k, s j}=0 .
$$

The plane strain deformation

$$
u_{1}=u_{1}\left(x_{1}, x_{2}\right), u_{2}=u_{2}\left(x_{1}, x_{2}\right), u_{3}=0
$$

and the antiplane strain deformation

$$
u_{1}=u_{2}=0, u_{3}=u_{3}\left(x_{1}, x_{2}\right)
$$

are decoupled provided (Ting 1995)

$$
C_{14}=C_{15}=C_{24}=C_{25}=C_{46}=C_{56}=0 .
$$

In equation (6), we have used the contracted Voigt notation for the stiffnesses $C_{i j k s}$ according to the scheme

$11 \rightarrow 1,22 \rightarrow 2,33 \rightarrow 3,23 \rightarrow 4,13 \rightarrow 5,12 \rightarrow 6$. The conditions (6) are satisfied by a monoclinic material with $x_{3}=0$ as the symmetry plane. However, equation (6) represents a material more general than a monoclinic material because the latter requires $C_{34}=C_{35}=0$ also. Assuming that the conditions (6) are satisfied, the equations of equilibrium (3) for antiplane strain reduce to a single equation

$$
C_{55} u_{3,11}+2 C_{45} u_{3,12}+C_{44} u_{3,22}=0 .
$$

In the following it will be assumed that the anisotropic material under discussion satisfies the relations (6). However, we shall refer to the material as monoclinic.

\section{Line force}

As shown by Ting (1995), the solution of equation (7) representing the displacement field due to a line force $f$ per unit length parallel to the $x_{3}$-axis acting in an infinite, homogeneous, monoclinic, elastic medium at the point $x_{1}=0, x_{2}=d$ is

$$
u_{3}=-\frac{f}{2 \pi m} \operatorname{Re} \ln (z-p d),
$$

where Re denotes the real part and

$$
\begin{aligned}
z & =x_{1}+p x_{2}, \\
p & =\left(-C_{45}+i m\right) / C_{44}, i=\sqrt{ }-1, \\
m & =\left(C_{44} C_{55}-C_{45}^{2}\right)^{1 / 2}>0 .
\end{aligned}
$$

The corresponding stresses are given by

$$
\tau_{31}=-\phi_{, 2}, \tau_{32}=\phi_{, 1},
$$

where

$$
\phi=\frac{f}{2 \pi} \operatorname{Im} \ln (z-p d)
$$

and $I m$ indicates the imaginary part.

The elastic field due to a line force $f$ placed at the point $x_{1}=0, x_{2}=d$ of a monoclinic halfspace $\left(M_{1} ; x_{2}>0\right)$ in welded contact with another monoclinic half-space $\left(M_{2} ; x_{2}<0\right)$ is given by (Ting 1995)

$$
\begin{aligned}
u_{3}^{(1)}= & -\frac{f}{2 \pi m^{(1)}} \operatorname{Re}\left\{\ln \left[z^{(1)}-p^{(1)} d\right]\right. \\
& \left.-K \ln \left[z^{(1)}-\bar{p}^{(1)} d\right]\right\}, \\
\phi^{(1)}= & \frac{f}{2 \pi} \operatorname{Im}\left\{\ln \left[z^{(1)}-p^{(1)} d\right]\right. \\
& \left.-K \ln \left[z^{(1)}-\bar{p}^{(1)} d\right]\right\}, \\
u_{3}^{(2)}= & -\frac{f(1+K)}{2 \pi m^{(2)}} \operatorname{Re} \ln \left[z^{(2)}-p^{(1)} d\right], \\
\phi^{(2)}= & \frac{f(1+K)}{2 \pi} \operatorname{Im} \ln \left[z^{(2)}-p^{(1)} d\right],
\end{aligned}
$$

where an overbar denotes complex conjugate and

$$
\begin{aligned}
K & =\frac{m^{(2)}-m^{(1)}}{m^{(2)}+m^{(1)}}, \quad(-1<K<1), \\
z^{(n)} & =x_{1}+p^{(n)} x_{2}, \quad(n=1,2), \\
p^{(n)} & =\left[-C_{45}^{(n)}+i m^{(n)}\right] / C_{44}^{(n)}, \\
m^{(n)} & =\left[C_{44}^{(n)} C_{55}^{(n)}-C_{45}^{(n)^{2}}\right]^{1 / 2} .
\end{aligned}
$$

For the half-space $M_{1}\left(x_{2}>0\right), C_{i j}^{(1)}$ are the stiffnesses, $u_{3}^{(1)}$ is the horizontal displacement and $\phi^{(1)}$ is the potential function giving the stresses through equation (10). A similar notation is used for the half-space $M_{2}\left(x_{2}<0\right)$. The solution given by equations (12) to (15) satisfies the following boundary conditions

$$
u_{3}^{(1)}=u_{3}^{(2)}, \tau_{32}^{(1)}=\tau_{32}^{(2)} \text { at } x_{2}=0 .
$$




\section{Strike-slip fault}

Taking the $x_{3}$-axis along the strike of the fault and the $x_{2}$-axis vertically downwards, the displacement field due to a long strike-slip fault of arbitrary orientation can be expressed as the line integral (Maruyama 1966)

$$
u_{3}(\mathbf{x})=\int_{L} \Delta u_{3}(\boldsymbol{\xi}) G_{3 k}^{3}(\mathbf{x}, \boldsymbol{\xi}) n_{k}(\boldsymbol{\xi}) d s(\boldsymbol{\xi}),
$$

where $\Delta u_{3}(\xi)$ is the displacement discontinuity, $n_{k}$ is the unit normal to the fault section $L$ and

$$
G_{3 k}^{3}(\mathbf{x}, \boldsymbol{\xi})=C_{3 k 3 s} \frac{\partial}{\partial \xi_{\mathrm{s}}} G_{3}^{3}(\mathbf{x}, \boldsymbol{\xi}) .
$$

In equation (22), $G_{3}^{3}(\mathbf{x}, \boldsymbol{\xi})$ is the Green's function representing the displacement at the point $(\mathbf{x})$ in the $x_{3}$-direction due to a line force of unit magnitude acting at the point $(\boldsymbol{\xi})$ in the $x_{3}$-direction. From equations (12) and (14), we have, for a line force of unit magnitude acting at the point $(\xi)$ in the $x_{3}$-direction placed in the monoclinic half-space $M_{1}\left(x_{2}>0\right)$ in welded contact with a dissimilar monoclinic half-space $M_{2}\left(x_{2}<0\right)$,

$$
\begin{aligned}
G_{3}^{3(1)}= & -\frac{1}{2 \pi m^{(1)}} \operatorname{Re}\left\{\ln \left[x_{1}-\xi_{1}+p^{(1)}\left(x_{2}-\xi_{2}\right)\right]\right. \\
& \left.-K \ln \left[x_{1}-\xi_{1}+p^{(1)} x_{2}-\bar{p}^{(1)} \xi_{2}\right]\right\} \\
= & -\frac{1}{2 \pi m^{(1)}}\left(\ln R_{1}-K \ln S_{1}\right),\left(x_{2}>0\right),
\end{aligned}
$$

$$
\begin{aligned}
G_{3}^{3(2)} & =-\frac{1+K}{2 \pi m^{(2)}} \operatorname{Re} \ln \left[x_{1}-\xi_{1}+p^{(2)} x_{2}-p^{(1)} \xi_{2}\right] \\
& =-\frac{1+K}{2 \pi m^{(2)}} \ln R_{2}, \quad\left(x_{2}<0\right),
\end{aligned}
$$

where

$$
\begin{aligned}
R_{1}^{2}= & {\left[x_{1}-\xi_{1}+\epsilon_{1}\left(x_{2}-\xi_{2}\right)\right]^{2}+\left[\alpha_{1}\left(x_{2}-\xi_{2}\right)\right]^{2}, } \\
S_{1}^{2}= & {\left[x_{1}-\xi_{1}+\epsilon_{1}\left(x_{2}-\xi_{2}\right)\right]^{2}+\left[\alpha_{1}\left(x_{2}+\xi_{2}\right)\right]^{2}, } \\
R_{2}^{2}= & \left(x_{1}-\xi_{1}+\epsilon_{2} x_{2}-\epsilon_{1} \xi_{2}\right)^{2} \\
& +\left(\alpha_{2} x_{2}-\alpha_{1} \xi_{2}\right)^{2}, \\
p^{(n)}= & \epsilon_{n}+i \alpha_{n}, \bar{p}^{(n)}=\epsilon_{n}-i \alpha_{n}, \quad(n=1,2), \\
\epsilon_{n}= & -C_{45}^{(n)} / C_{44}^{(n)}, \alpha_{n}=\left(\gamma_{n}-\epsilon_{n}^{2}\right)^{1 / 2}=m^{(n)} / C_{44}^{(n)}, \\
\gamma_{n}= & C_{55}^{(n)} / C_{44}^{(n)} .
\end{aligned}
$$

Using the Voigt notation for the stiffnesses, equations (21) and (22) yield

$$
\begin{aligned}
u_{3}^{(k)}(\mathbf{x})= & \int_{\mathrm{L}} b\left\{\left[n_{1} C_{55}^{(1)}+n_{2} C_{45}^{(1)}\right] \frac{\partial}{\partial \xi_{1}}+\left[n_{1} C_{45}^{(1)}\right.\right. \\
& \left.\left.+n_{2} C_{44}^{(1)}\right] \frac{\partial}{\partial \xi_{2}}\right\} G_{3}^{3(k)}(\mathbf{x}, \boldsymbol{\xi}) d s \\
& (k=1,2),
\end{aligned}
$$

where $b=\Delta u_{3}^{(1)}$ is the displacement discontinuity. Inserting the expressions for $G_{3}^{3(1)}$ and $G_{3}^{3(2)}$ from equations (23) and (24), we find

$$
\begin{aligned}
u_{3}^{(1)}(\mathbf{x})= & \frac{1}{2 \pi m^{(1)}} \int_{\mathrm{L}} b\left\{[ n _ { 1 } C _ { 5 5 } ^ { ( 1 ) } + n _ { 2 } C _ { 4 5 } ^ { ( 1 ) } ] \left[x_{1}-\xi_{1}\right.\right. \\
& \left.+\epsilon_{1}\left(x_{2}-\xi_{2}\right)\right]\left(\frac{1}{R_{1}^{2}}-\frac{K}{S_{1}^{2}}\right) \\
& +\left[n_{1} C_{45}^{(1)}+n_{2} C_{44}^{(1)}\right] \times\left[\epsilon _ { 1 } \left\{x_{1}-\xi_{1}\right.\right. \\
& \left.+\epsilon_{1}\left(x_{2}-\xi_{2}\right)\right\}\left(\frac{1}{R_{1}^{2}}-\frac{K}{S_{1}^{2}}\right) \\
& \left.\left.+\alpha_{1}^{2}\left(\frac{x_{2}-\xi_{2}}{R_{1}^{2}}+K \frac{x_{2}+\xi_{2}}{S_{1}^{2}}\right)\right]\right\} d s, \\
u_{3}^{(2)}(\mathbf{x})= & \frac{1+K}{2 \pi m^{(2)}} \int_{\mathrm{L}} b\left\{[ n _ { 1 } C _ { 5 5 } ^ { ( 1 ) } + n _ { 2 } C _ { 4 5 } ^ { ( 1 ) } ] \left(x_{1}-\xi_{1}\right.\right. \\
& \left.+\epsilon_{2} x_{2}-\epsilon_{1} \xi_{2}\right)+\left[n_{1} C_{45}^{(1)}+n_{2} C_{44}^{(1)}\right] \\
& \times\left[\epsilon_{1}\left(x_{1}-\xi_{1}+\epsilon_{2} x_{2}-\epsilon_{1} \xi_{2}\right)\right. \\
& \left.\left.+\alpha_{1}\left(\alpha_{2} x_{2}-\alpha_{1} \xi_{2}\right)\right]\right\} \frac{1}{R_{2}^{2}} d s .
\end{aligned}
$$

Consider a strike-slip fault of width $L$ and infinite length along the strike $\left(x_{3}\right)$-direction. Let $d$ be the distance of the upper edge $A$ of the fault from the interface. If $(s, \delta)$ are the polar coordinates of any point $Q\left(\xi_{1}, \xi_{2}\right)$ on the fault with $A$ as the origin, we have (figure 1 )

$$
\begin{aligned}
\xi_{1} & =s \cos \delta, & \xi_{2} & =d+s \sin \delta, \\
n_{1} & =-\sin \delta, & n_{2} & =\cos \delta .
\end{aligned}
$$

Using these values and (25), equations (27) and (28) yield

$$
u_{3}^{(1)}(\mathbf{x})=\frac{\alpha_{1}}{2 \pi} \int_{0}^{\mathrm{L}}\left(\frac{Y_{5}}{R_{1}^{2}}+K \frac{Y_{6}}{S_{1}^{2}}\right) b d s,
$$




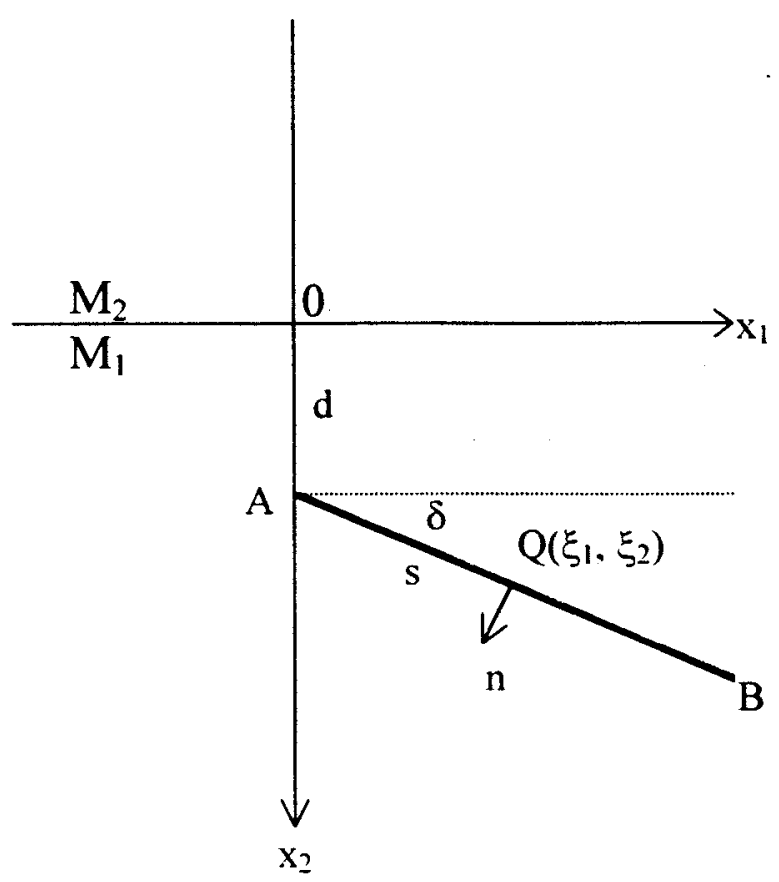

Figure 1. Geometry of a long fault in a half-space $\left(M_{1}\right)$ in welded contact with a dissimilar half-space $\left(M_{2}\right)$. The fault is of infinite length in the strike $\left(x_{3}\right)$ - direction. $A B$ is the fault section by the $x_{1} x_{2}$ - plane, which is also the plane of elastic symmetry of the monoclinic elastic media, $d$ is the distance of the upper edge $A$ of the fault from the interface and $\delta$ the dip angle. $(s, \delta)$ denote the polar coordinates of any point $Q\left(\xi_{1}, \xi_{2}\right)$ on the fault.

$$
u_{3}^{(2)}(\mathbf{x})=\frac{1-K}{2 \pi} \int_{0}^{\mathrm{L}} \frac{Y_{7}}{R_{2}^{2}} b d s,
$$

where

$$
\begin{aligned}
R_{1}^{2}= & \left(A+\epsilon_{1} \sin 2 \delta\right) s^{2}-2\left[C x_{1}+B\left(x_{2}-d\right)\right] s \\
& +x_{1}^{2}+\gamma_{1}\left(x_{2}-d\right)^{2}+2 \epsilon_{1} x_{1}\left(x_{2}-d\right) \\
= & \frac{1}{A+\epsilon_{1} \sin 2 \delta}\left\{\left[\left(A+\epsilon_{1} \sin 2 \delta\right) s-C x_{1}\right.\right. \\
& \left.\left.-B\left(x_{2}-d\right)\right]^{2}+\alpha_{1}^{2} Y_{5}^{2}\right\},
\end{aligned}
$$$$
S_{1}^{2}=\left(A+\epsilon_{1} \sin 2 \delta\right) s^{2}-2\left[C\left(x_{1}+2 \epsilon_{1} x_{2}\right)\right.
$$$$
\left.-B\left(x_{2}+d\right)\right] s+x_{1}^{2}+\gamma_{1}\left(x_{2}+d\right)^{2}
$$$$
+2 \epsilon_{1} x_{1}\left(x_{2}-d\right)-4 \epsilon_{1}^{2} x_{2} d
$$$$
=\frac{1}{A+\epsilon_{1} \sin 2 \delta}\left\{\left[\left(A+\epsilon_{1} \sin 2 \delta\right) s\right.\right.
$$$$
\left.\left.-C\left(x_{1}+2 \in_{1} x_{2}\right)+B\left(x_{2}+d\right)\right]^{2}+\alpha_{1}^{2} Y_{6}^{2}\right\},
$$$$
R_{2}^{2}=\left(A+\epsilon_{1} \sin 2 \delta\right) s^{2}-2\left[C x_{1}+\left(C \in_{2}\right.\right.
$$$$
\left.\left.+\alpha_{1} \alpha_{2} \sin \delta\right) x_{2}-B d\right] s+x_{1}^{2}+\gamma_{2} x_{2}^{2}+\gamma_{1} d^{2}
$$$$
+2 \epsilon_{2} x_{1} x_{2}-2 \epsilon_{1} x_{1} d-2\left(\alpha_{1} \alpha_{2}+\epsilon_{1} \epsilon_{2}\right) d x_{2}
$$

$$
\begin{aligned}
= & \frac{1}{A+\epsilon_{1} \sin 2 \delta}\left\{\left[\left(A+\epsilon_{1} \sin 2 \delta\right) s\right.\right. \\
& \left.\left.-C x_{1}-\left(C \in_{2}+\alpha_{1} \alpha_{2} \sin \delta\right) x_{2}+B d\right]^{2}+Y_{7}^{2}\right\},
\end{aligned}
$$

$Y_{5}=-x_{1} \sin \delta+\left(x_{2}-d\right) \cos \delta$,

$Y_{6}=\left(x_{1}+2 \in_{1} x_{2}\right) \sin \delta+\left(x_{2}+d\right) \cos \delta$,

$Y_{7}=-\alpha_{1} x_{1} \sin \delta+x_{2}\left[\alpha_{2} \cos \delta\right.$

$$
\left.-\left(\alpha_{1} \epsilon_{2}-\alpha_{2} \epsilon_{1}\right) \sin \delta\right]-\alpha_{1} \cos \delta d \text {, }
$$

$A=\cos ^{2} \delta+\gamma_{1} \sin ^{2} \delta$,

$B=\epsilon_{1} \cos \delta+\gamma_{1} \sin \delta$,

$C=\cos \delta+\epsilon_{1} \sin \delta$.

Assuming $b$ to be constant over $L$ and performing the integration in equations (30) and (31), we obtain

$$
\begin{aligned}
& u_{3}^{(1)}(\mathbf{x})=\frac{b}{2 \pi} \\
& \times \tan ^{-1}\left[\frac{\left(A+\epsilon_{1} \sin 2 \delta\right) L-C x_{1}-B\left(x_{2}-d\right)}{\alpha_{1}\left\{\left(x_{2}-d\right) \cos \delta-x_{1} \sin \delta\right\}}\right] \\
& +\frac{b}{2 \pi} \tan ^{-1}\left[\frac{C x_{1}+B\left(x_{2}-d\right)}{\alpha_{1}\left\{\left(x_{2}-d\right) \cos \delta-x_{1} \sin \delta\right\}}\right] \\
& +\frac{b K}{2 \pi}\left[\begin{array}{c}
\left(A+\epsilon_{1} \sin 2 \delta\right) L-C\left(x_{1}+2 \in \epsilon_{1}\right) \\
\alpha_{1}\left\{\left(x_{1}+2 \in \epsilon_{1} x_{2}\right) \sin \delta\right. \\
\left.+\left(x_{2}+d\right) \cos \delta\right\}
\end{array}\right] \\
& \times \tan ^{-1}\left[\frac{b K}{2 \pi}\right] \\
& \times \tan ^{-1}\left[\frac{C\left(x_{1}+2 \in_{1} x_{2}\right)-B\left(x_{2}+d\right)}{\alpha_{1}\left\{\left(x_{1}+2 \epsilon_{1} x_{2}\right) \sin \delta+\left(x_{2}+d\right) \cos \delta\right\}}\right]
\end{aligned}
$$

$$
\begin{aligned}
& u_{3}^{(2)}(\mathbf{x})= \frac{(1-K) b}{2 \pi} \\
& \times \tan ^{-1}\left[\begin{array}{r}
\left(A+\epsilon_{1} \sin 2 \delta\right) L-C x_{1}-\left(C \in_{2}\right. \\
\left.+\alpha_{1} \alpha_{2} \sin \delta\right) x_{2}+B d
\end{array}\right. \\
&\left.\begin{array}{r}
\left.\alpha_{2} \cos \delta-\left(\alpha_{1} \epsilon_{2}-\alpha_{2} \epsilon_{1}\right) \sin \delta\right\} x_{2} \\
-\alpha_{1} x_{1} \sin \delta-\alpha_{1} \cos \delta d
\end{array}\right]
\end{aligned}
$$$$
+\frac{(1-K) b}{2 \pi}
$$

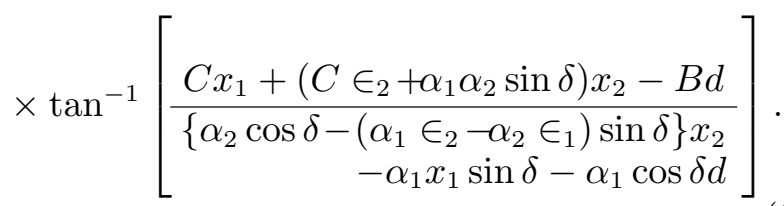




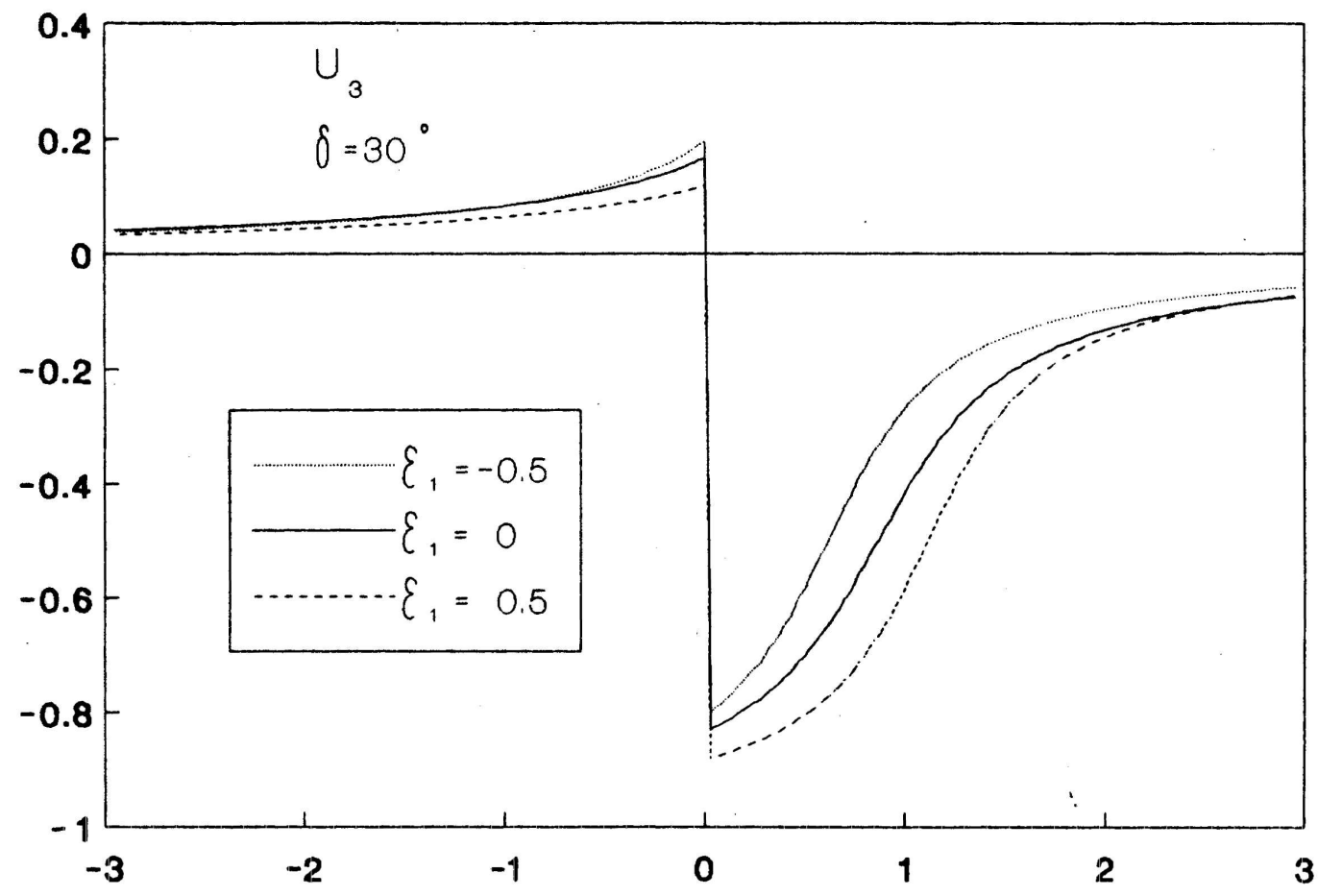

Figure 2(a). Variation of the dimensionless along-strike surface displacement $\left(U_{3}\right)$ with the dimensionless horizontal distance $\left(x_{1} / L\right)$ from the upper edge of the fault for $\gamma_{1}=1$ and $\epsilon_{1}=0,+0.5,-0.5$. The fault is situated in a uniform monoclinic elastic half-space. The dip angle $\delta$ is (a) $30^{\circ}$, (b) $60^{\circ}$, (c) $90^{\circ}$.

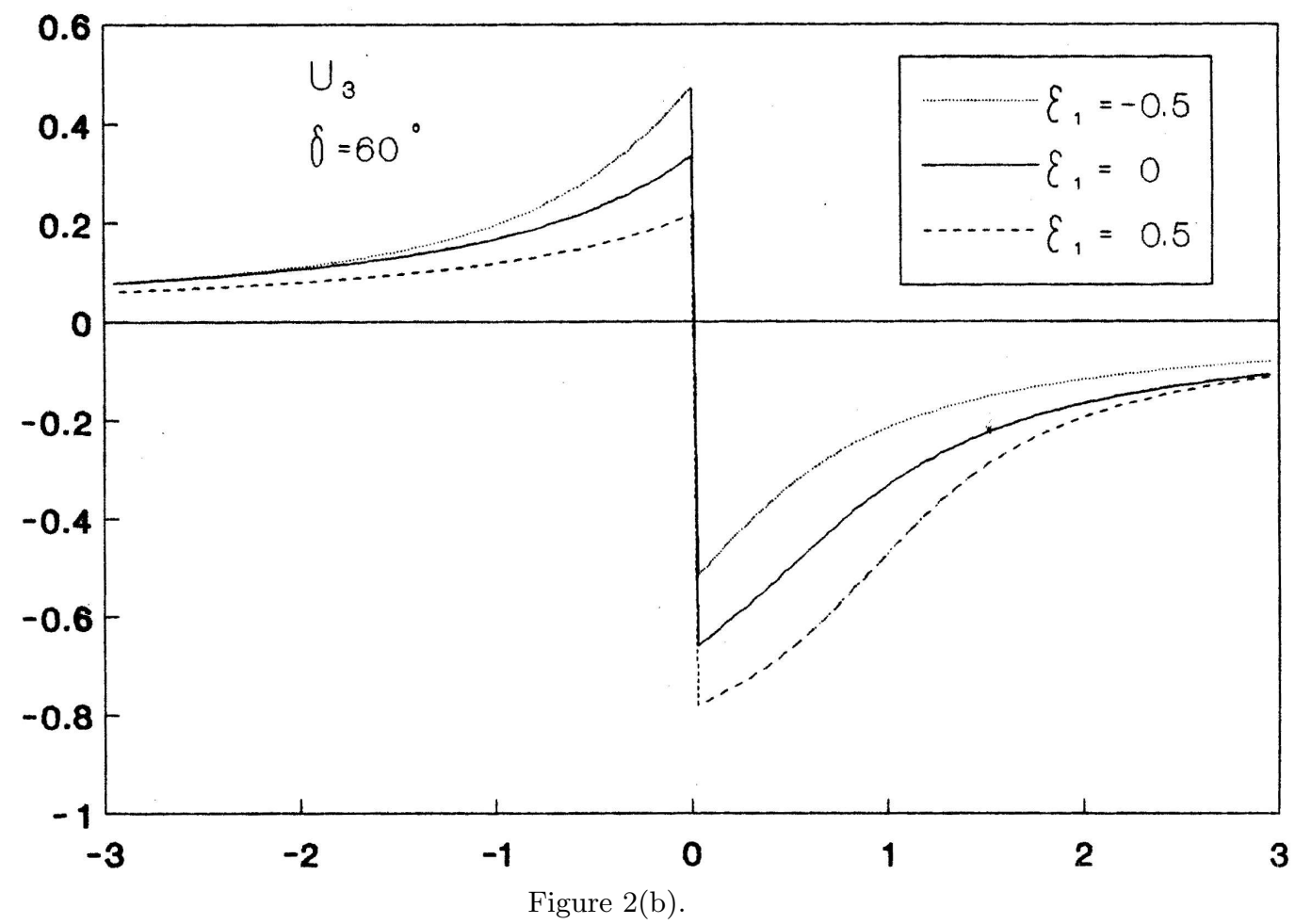

Equations (33) and (34) yield the displacement field due to a long inclined strike-slip fault in a monoclinic half-space in welded contact with a dissimilar monoclinic half-space. On taking $K=-1$ in equation (33), we obtain the displacement field in a monoclinic half-space with a free boundary.
These results coincide with the corresponding results obtained by Singh et al (2001). Similarly, on taking $K=1$, we obtain the displacement field in a monoclinic half-space with a rigid boundary. The field in an unbounded monoclinic medium is recovered on taking $K=0$. 

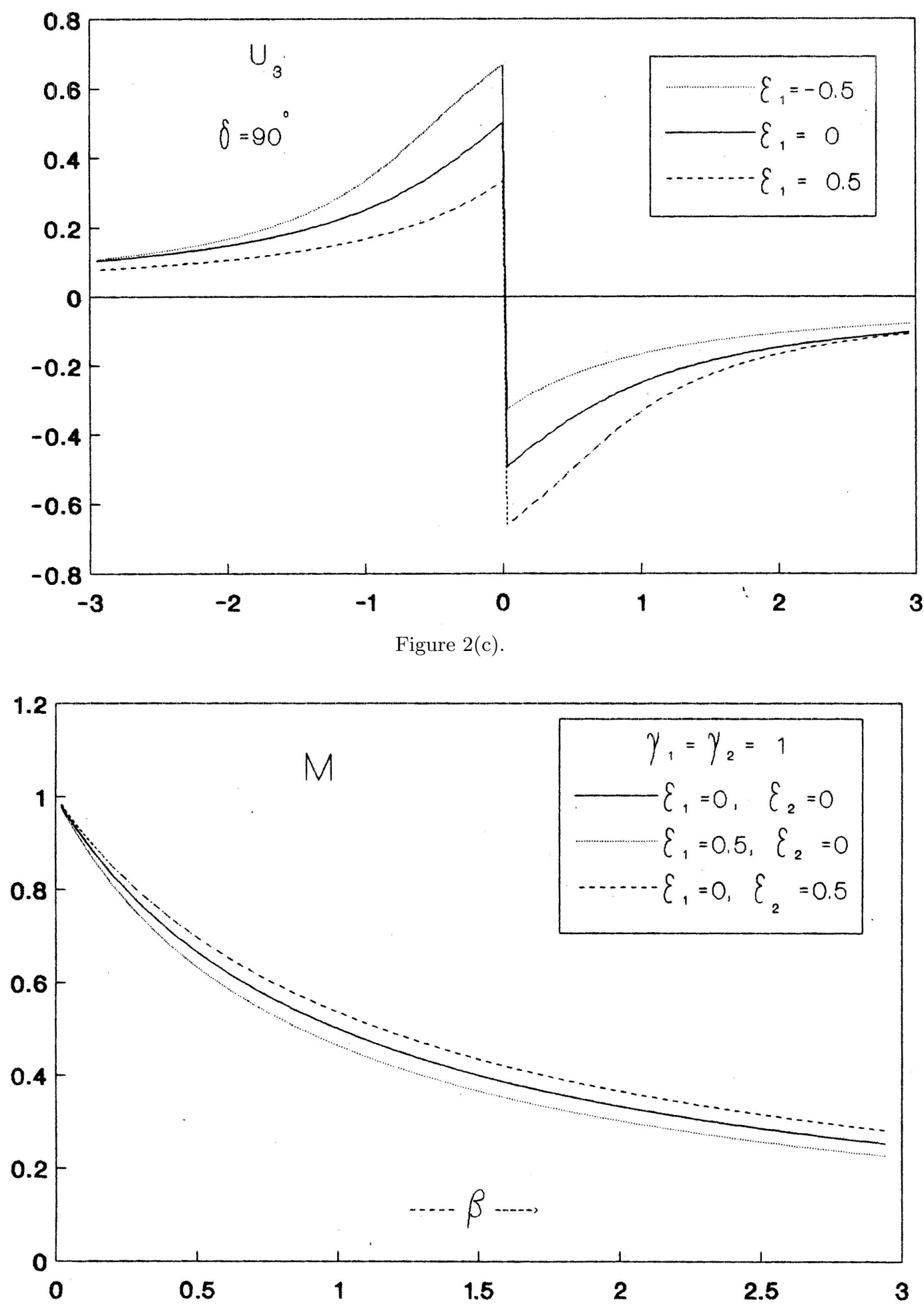

Figure 3. Variation of the magnification factor $M$ with the rigidity contrast $\beta=C_{44}^{(2)} / C_{44}^{(1)}$ for $\left|\in_{1}\right|=0,0.5$ and $\left|\epsilon_{2}\right|=0,0.5$.

For an isotropic material, $\epsilon_{1}=\epsilon_{2}=0, \gamma_{1}=\gamma_{2}=1$, $m^{(1)}=\mu_{1}, m^{(2)}=\mu_{2}$ and equations (33) and (34) become

$$
u_{3}^{(1)}(\mathbf{x})=\frac{b}{2 \pi} \tan ^{-1}\left[\frac{L-x_{1} \cos \delta-\left(x_{2}-d\right) \sin \delta}{\left(x_{2}-d\right) \cos \delta-x_{1} \sin \delta}\right]
$$

$+\frac{b}{2 \pi} \tan ^{-1}\left[\frac{x_{1} \cos \delta+\left(x_{2}-d\right) \sin \delta}{\left(x_{2}-d\right) \cos \delta-x_{1} \sin \delta}\right]$ $+\frac{b}{2 \pi}\left(\frac{\mu_{2}-\mu_{1}}{\mu_{2}+\mu_{1}}\right)\left\{\tan ^{-1}\left[\frac{L-x_{1} \cos \delta+\left(x_{2}+d\right) \sin \delta}{\left(x_{2}+d\right) \cos \delta+x_{1} \sin \delta}\right]\right.$ $\left.+\tan ^{-1}\left[\frac{x_{1} \cos \delta-\left(x_{2}+d\right) \sin \delta}{\left(x_{2}+d\right) \cos \delta+x_{1} \sin \delta}\right]\right\}$, 


$$
\begin{aligned}
& u_{3}^{(2)}(\mathbf{x}) \\
& \quad=\frac{b \mu_{1}}{\pi\left(\mu_{1}+\mu_{2}\right)}\left\{\tan ^{-1}\left[\frac{L-x_{1} \cos \delta-\left(x_{2}-d\right) \sin \delta}{\left(x_{2}-d\right) \cos \delta-x_{1} \sin \delta}\right]\right. \\
& \left.+\tan ^{-1}\left[\frac{x_{1} \cos \delta+\left(x_{2}-d\right) \sin \delta}{\left(x_{2}-d\right) \cos \delta-x_{1} \sin \delta}\right]\right\},
\end{aligned}
$$

where $\mu_{1}$ and $\mu_{2}$ are the rigidities of the two media. Equations (35) and (36) are in agreement with the corresponding results of Rani and Singh (1993) for the displacement field due to a long inclined strike-slip fault in an isotropic half-space in welded contact with another isotropic half-space for the particular case $d=0$.

\section{Numerical results}

The displacement is continuous across the interface $x_{2}=0$. Taking $d=0, x_{2}=0$, equations (33) and (34) yield the following expression for the horizontal displacement at the interface

$$
u_{3}^{(1)}=u_{3}^{(2)}=b M U_{3}
$$

where

$$
\begin{aligned}
U_{3}= & -\frac{1}{\pi}\left\{\tan ^{-1}\left[\frac{\left(A+\epsilon_{1} \sin 2 \delta\right) L-C x_{1}}{\alpha_{1} x_{1} \sin \delta}\right]\right. \\
& \left.+\tan ^{-1}\left[\frac{C}{\alpha_{1} \sin \delta}\right]\right\},
\end{aligned}
$$$$
M=(1-K) / 2=\frac{1}{1+\left(\alpha_{2} / \alpha_{1}\right) \beta}, \beta=C_{44}^{(2)} / C_{44}^{(1)} .
$$

From equation (38), we note that $U_{3}$ depends only on the elastic properties of the source half-space; it is independent of the elastic properties of the other half-space. In fact, $U_{3}$ represents the dimensionless displacement at the free surface of a monoclinic half-space caused by a surface-breaking long inclined strike-slip fault. The effect of the other half-space on the displacement at the interface between two welded half-spaces is to introduce the magnification factor $M$. The value of $M$ is 1 for a uniform half-space with a free surface and $1 / 2$ for a uniform whole space.

Figure 2 shows the variation of $U_{3}$ with the dimensionless horizontal distance $x_{1} / L$ from the upper edge of the fault for $\gamma_{1}=0$ and $\epsilon_{1}=$ $0, \pm 0.5$, for three values of the dip angle $\delta=$ $30^{\circ}, 60^{\circ}, 90^{\circ} . \epsilon_{1}=0$ corresponds to the isotropic medium. Figure 3 shows the variation of the magnification factor $M$ with the rigidity contrast $\beta$, for $\gamma_{1}=\gamma_{2}=1,\left|\epsilon_{1}\right|=0,0.5,\left|\epsilon_{2}\right|=0,0.5$. We note that the anisotropy parameter of the source half-space $\left(\epsilon_{1}\right)$ has a significant effect on the displacement field. However, the effect of the anisotropy parameter of the other half-space $\left(\epsilon_{2}\right)$ is small. The rigidity contrast $(\beta)$ has a strong influence on the displacement field.

\section{Acknowledgement}

The authors are thankful to the Council of Scientific and Industrial Research, New Delhi for financial support through the Emeritus Scientist Scheme awarded to SJS.

\section{References}

Crampin S 1989 Suggestions for a consistent terminology for seismic anisotropy; Geophys. Prospect. 37 753-770

Garg N R, Madan D K and Sharma R K 1996 Two-dimensional deformation of an orthotropic elastic medium due to seismic sources; Phys. Earth Planet. Inter. 94 43-62

Maruyama T 1966 On two-dimensional elastic dislocations in an infinite and semi-infinite medium; Bull. Earthq. Res. Inst. 44 811-871

Pan E 1989 Static response of a transversely isotropic layered half-space to general dislocation sources Phys. Earth Planet. Inter. 58 103-117

Rani S and Singh S J 1993 Residual elastic field in two welded half-spaces caused by a long inclined strikeslip fault; Proc. Indian natn. Sci. Acad. $59 \quad 455-$ 464

Savage J C 1980 Dislocations in seismology; In: Dislocations in solids Vol. 3 Moving dislocations (ed) F R N Nabarro (Amsterdam : North-Holland Publishing Co.) pp. 251-339

Sharma R K, Garg N R and Singh K 1991 Static deformation of two welded half-spaces due to a very long strikeslip dislocation; Proc. Indian Acad. Sci. (Earth Planet. Sci.) $100379-388$

Singh S J, Kumar A and Singh J 2001 Deformation of a monoclinic elastic half-space by a long inclined strike-slip fault (submitted for publication)

Ting T C T 1995 Antiplane deformations of anisotropic elastic material; In: Recent advances in elasticity, viscoelasticity and inelasticity, Series in Advances in mathematics in applied sciences Vol. 26 (ed) K R Rajgopal (Singapore : World Scientific) pp. $150-179$ 